\title{
Velocity autocorrelation function in uniformly heated granular gas
}

\author{
Shikha Kumari ${ }^{1, \star}$ and Syed Rashid Ahmad ${ }^{1, \star \star}$ \\ ${ }^{1}$ Department of Physics, Jamia Millia Islamia, New Delhi 110025, India
}

\begin{abstract}
In this paper we study aging of the velocity autocorrelation function of a uniformly heated granular gas using large scale event-driven molecular dynamics simulations in both 2 and 3 dimensional system. The system is heated by adding Gaussian white noise to each velocity component of all the particles. After a few collisions per particle, the system attains steady state. During early stages, the velocity autocorrelation function shows aging as there is explicit dependence of the function on $\tau_{w}$ but after steady state is reached, the velocity autocorrelation function become independent of $\tau_{w}$ and does not show any aging. Velocity correlations develop even after steady state is reached, these correlations are less pronounced in 3 dimensional system.
\end{abstract}

\section{Introduction}

A Granular material is a collection of mesoscopic particles called grains. These materials are abundant in the universe in different shapes with sizes ranging from powders to huge asteroids. Depending on the average kinetic energy of the individual grains, the system of granular matter can exhibit properties of solid, liquid or gas [1]. These materials are enriched with variety of phenomena when they are considered to be solid, liquid or gas $[1,2]$. The most important feature of the granular matter is that the constituent grains lose energy upon collision i.e. collisions are inelastic, thus leading to dissipation of energy. This inelastic property of granular matter causes variety of interesting phenomena such as pattern formation, clustering, jamming, size separation, etc in the system [1-4]. Some examples of natural processes involving granular matter are formation of dunes and other patterns in deserts, earthquakes, landslides, erosion etc [2].

In this study, we are considering granular gas [5-7], i.e. a granular system in which grains move separately and are not in contact with each other. Technically, granular gas is a dilute system in which the mean free path of particles is much greater than average particle size. The grains interact when they collide or overlap and these interactions are dissipative because of which they lose energy. If no energy is injected into the system, the system continue to lose energy and settles in an inhomogeneous cooling state $[7,8]$ where particle-rich clusters emerge in the system and within the clusters particles move in parallel directions. For studies related to these phenomena see Ref. [3, 9-11]. If energy is injected into the system to compensate energy loss due to collisions, the system settles to a non-equilibrium steady state. Energy is injected into the system through standard geometries like horizon-

\footnotetext{
^e-mail: k.shikha.physics@gmail.com
}

$\star \star$ e-mail: srahmad@jmi.ac.in tal or vertical vibrations [12, 13], rotation [14], shear [15], applying magnetic [16] or electrostatic [17] forces. For non-equilibrium systems, certain statistical quantities are history dependent i.e. they depend on both the time interval of measurement and the waiting time from where the measurement started. In case of granular gases, the velocity autocorrelation function exhibits this kind of explicit dependence on waiting time.

Earlier, a theoretical study of the velocity autocorrelation function of granular system was done in Ref.[18], Ahmad and Puri [8] have studied aging of the velocity autocorrelation function for a force free granular gas through numerical simulations. We aim at studying the velocity autocorrelation function for the forced case. In this work, we are considering uniformly heated granular gas. In previous work on heated systems, there have been studies on the velocity distributions in granular fluids [19], velocity correlations and distributions in granular gases [20, 21], velocity distributions in heated viscoelastic granular gas [22]. Till now no study has been done on the velocity autocorrelation function in heated systems. The velocity autocorrelation function is used to study history dependence in the system i.e. memory of previous behaviour. During each particle collision, local parallelization of particle velocities takes place i.e. correlations appear in velocity field. This correlated motion leads to formation of regions where particle density is more and also regions where density is less. The persistence of this domain growth is quantified by the velocity correlation and autocorrelation function. We study the behaviour of the velocity autocorrelation function in heated granular gas and compare the results with force free case. The system is subject to Gaussian white noise [19-21, 23, 24], where all particles are uniformly heated.

The paper is organised as follows. In Sec. 2, we briefly describe the system, i.e. we explain the equation of motion governing heated granular gas, how the particles veloci- 
ties update after interaction and what is the model used for heating the system. In Sec. 3, we report detailed simulation results from MD simulations and its discussion. Finally, in Sec. 4 we summarize and conclude the paper.

\section{Description of System}

The most important feature of granular matter is that the pairwise collisions are inelastic. The post-collision velocities are given in terms of pre-collision velocities as,

$$
\mathbf{v}_{i, j}^{\prime}=\mathbf{v}_{i, j} \mp \frac{1+\epsilon}{2}\left(\mathbf{v}_{i, j} \cdot \mathbf{e}\right) \mathbf{e}
$$

where $\mathbf{v}^{\prime}$ is post-collision velocity and $\mathbf{v}$ is pre-collision velocity, e is unit vector joining center of $i^{\text {th }}$ and $j^{\text {th }}$ particle at the time of collision and $\epsilon$ is called coefficient of restitution defined as,

$$
\epsilon=\left|\frac{\left(\mathbf{v}_{\mathrm{ij}}^{\prime} \cdot \mathbf{e}\right)}{\left(\mathbf{v}_{\mathrm{ij}} \cdot \mathbf{e}\right)}\right|
$$

where $v_{i j}$ and $v_{i j}^{\prime}$ are relative velocities of $i^{\text {th }}$ and $j^{\text {th }}$ particles before and after collision respectively. The kinetic energy of the granular system is defined as $E=\frac{\left\langle v^{2}\right\rangle}{d}$, where $\left\langle v^{2}\right\rangle$ is the mean-squared velocity and $\mathrm{d}$ is the dimension of the system.

\subsection{Uniformly Heated System}

The stochastic equation of motion for granular system driven by a white noise thermostat is described as,

$$
\frac{d \mathbf{v}_{i}}{d t}=\frac{\mathbf{F}_{i}^{c}}{m}+\frac{\mathbf{F}_{i}^{t}}{m}
$$

where $m$ is the mass of the particle, $\mathbf{F}_{i}^{c}$ is the force on the $i^{\text {th }}$ particle $(i=1,2, \ldots N)$ due to pairwise collision and $\mathbf{F}_{i}^{t}$ is the external force which is considered as Gaussian white noise with zero mean and is uncorrelated for different particles i.e,

$$
\begin{aligned}
& \left\langle F_{i, \alpha}^{t}(t) F_{j, \beta}^{t}\left(t^{\prime}\right)\right\rangle=\xi_{0}^{2} \delta_{i j} \delta_{\alpha \beta} \delta\left(t-t^{\prime}\right) \\
& \left\langle\mathbf{F}_{i}^{t}(t)\right\rangle=0
\end{aligned}
$$

where $\alpha, \beta=\{x, y, z\}, \xi_{0}$ characterizes the strength of stochastic force, $\delta_{i j}$ and $\delta_{\alpha \beta}$ are Kronecker delta and $\delta\left(t-t^{\prime}\right)$ is the delta function.

\subsection{The Velocity Autocorrelation Function}

The velocity autocorrelation function is defined as,

$$
A\left(\tau_{w}, \tau\right)=\frac{1}{N} \sum_{i=1}^{N}\left\langle\mathbf{v}_{\mathbf{i}}\left(\tau_{\mathbf{w}}\right) \cdot \mathbf{v}_{\mathbf{i}}(\tau)\right\rangle
$$

It measures correlations in the velocity of a particle in time. It is used to study history dependence i.e. the memory of previous behaviour of particles in the system. The autocorrelation function is calculated at collision time $\tau$

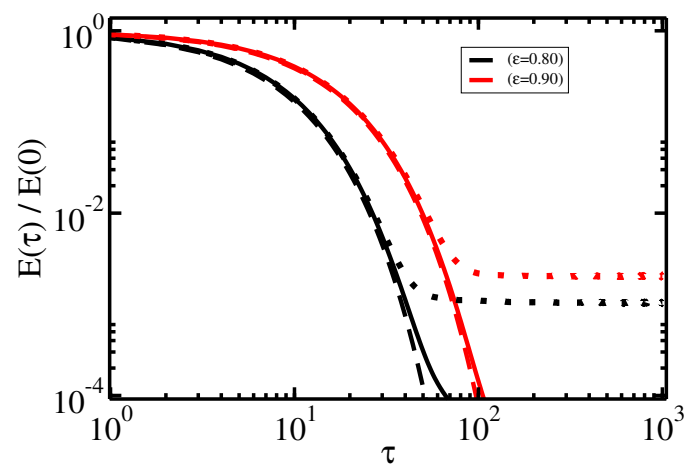

Figure 1. Time evolution of the normalised kinetic energy $E(\tau) / E(0)$ for force free and heated case for 2 dimensional system. Coefficient of restitution $\epsilon=0.80$ and 0.90 . Dotted lines represent simulation results for heated gas, solid lines represent simulation results for force free gas and dashed lines represent Haff's Law.

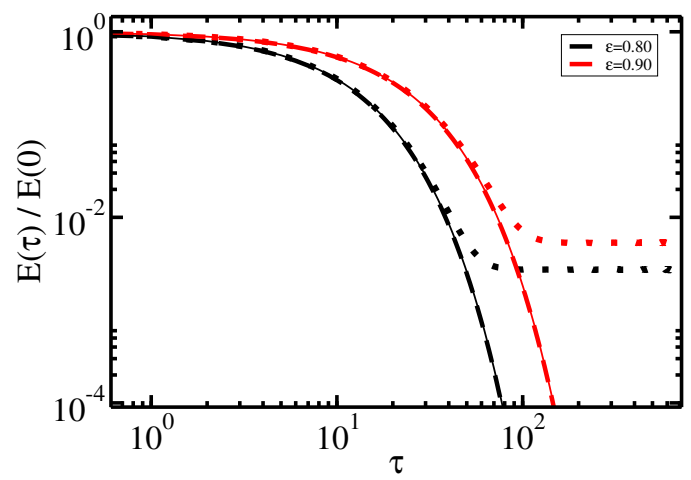

Figure 2. Time evolution of the normalised kinetic energy $E(\tau) / E(0)$ for heated case for 3 dimensional system. Coefficient of restitution $\epsilon=0.80$ and 0.90 . Dotted lines represent simulation results for heated gas, solid lines represent simulation results for force free gas and dashed lines represent Haff's Law.

with respect to reference or waiting time $\tau_{w}$. For an equilibrium system autocorrelation function depends on time difference $\tau-\tau_{w}$, but for a non-equilibrium system it depends explicitly on both $\tau$ and $\tau_{w}$ rather than their difference. This dependence of autocorrelation function on $\tau_{w}$ is called aging or history dependency. A cooling granular gas is a non-equilibrium system and therefore the corresponding velocity autocorrelation function exhibits aging properties.

\subsection{Simulation Details}

We use event driven molecular dynamics simulation [25] to study the heated granular system. The system consists of $N=100000$ particles having unit mass and unit diameter. We have simulated these particles in both 2dimensional and 3-dimensional box. Size of the box for both the cases is chosen such that the number density is 0.02 for both the cases and periodic boundary conditions are applied in all the directions. Since we are using event driven simulations the velocities of grains are updated according to Eq. (1), and during simulations we add heat 

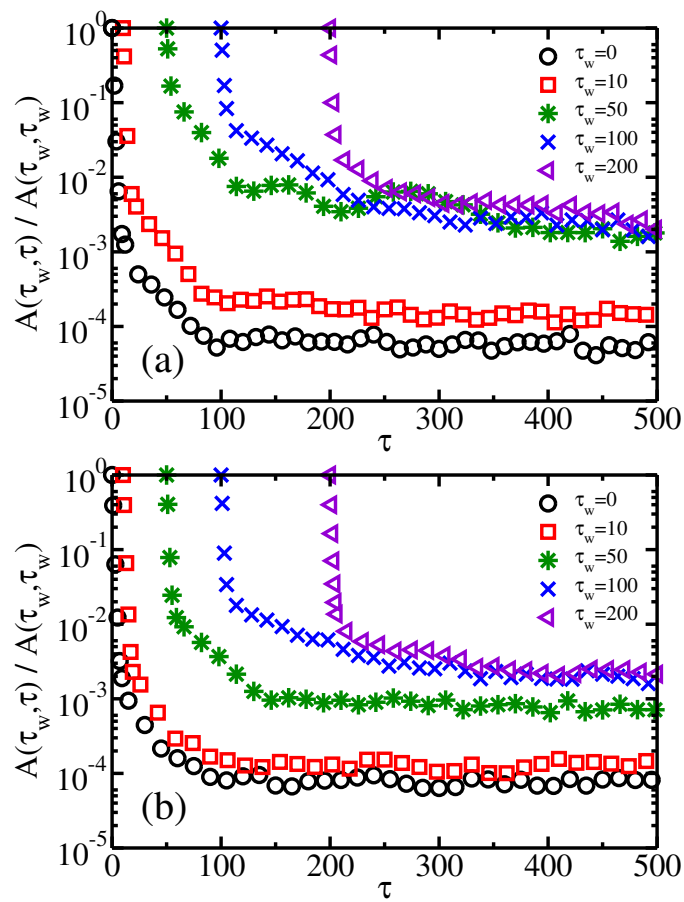

Figure 3. The velocity autocorrelation functions for 2 dimensional system with different reference time $\tau_{w}=$ $0,10,50,100,200$. Coefficient of restitution values taken are (a) $\epsilon=0.80$ and $(\mathrm{b}) \epsilon=0.90$.

component to velocity of each particle after every time step dt using following equation,

$$
v_{i}(t+d t)=v_{i}(t)+\sqrt{r} \sqrt{d t} \xi
$$

where $\xi$ is random variable which is uniformly distributed between $\left[-\frac{1}{2}, \frac{1}{2}\right], r$ is amplitude of noise. After adjusting the velocities, the system is shifted to center-of-mass frame,

$$
\mathbf{v}_{i}=\mathbf{v}_{i}-\frac{1}{N} \sum_{i=1}^{N} \mathbf{v}_{i}
$$

This ensures conservation of momentum and that the speed of the particles do not increase indefinitely. For convenience we have used a new time variable $\tau$, which represents the average number of collisions a particle has suffered ( $\tau=1$ implies one collision per particle). In our simulations we have used $r=0.1$ for 3-dimensional case and $r=10$ for 2-dimensional case. A high value of the noise amplitude $r$ is required in 2-dimensional case since, $r=0.1$ was not sufficient to overcome dissipation in the system. The system is initialised by randomly placing particles in the box using random number generator. Then, velocities are assigned to each particle randomly in such a way that $\sum_{i} v_{i}=0$. The kinetic energy $E$ and the aging behaviour of heated system is studied for different values of coefficient of restitution $(\epsilon=0.80,0.85,0.90)$. For a particular value of $\epsilon$ the system is evolved till collision time $\tau=1000$ and the velocity autocorrelation function is calculated according to Eq.(5) with respect to different reference times $\tau_{w}=0,10,50,100,200$. All statistical quantities are averaged over 30 independent runs.
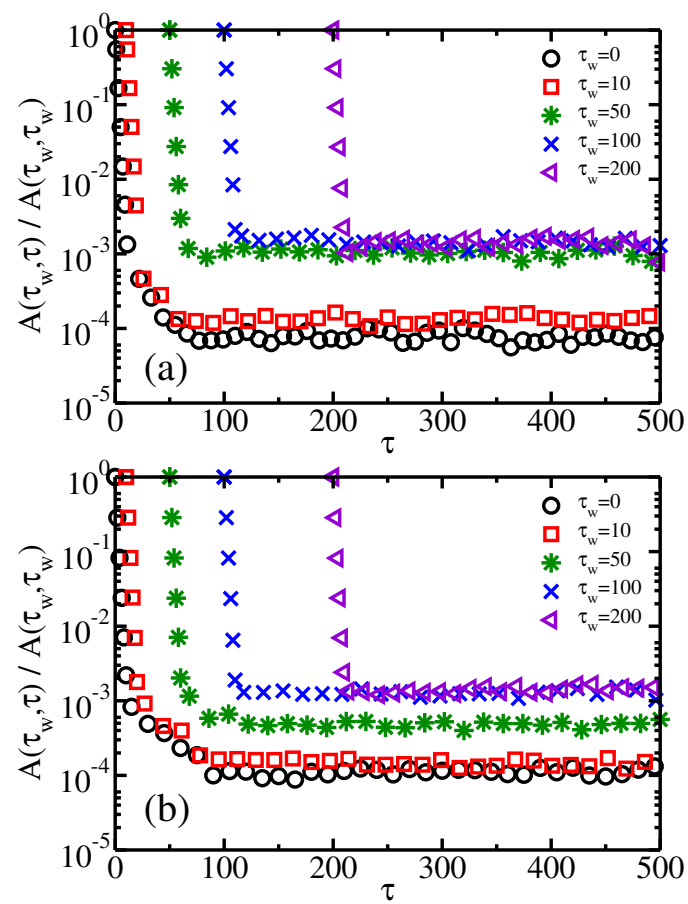

Figure 4. The velocity autocorrelation functions for 3 dimensional system with different reference time $\tau_{w}=$ $0,10,50,100,200$. Coefficient of restitution values taken are (a) $\epsilon=0.80$ and (b) $\epsilon=0.90$.

\section{Results and Discussion}

In Fig.1, time evolution of the normalised kinetic energy of granular gas is shown on log-log scale for a 2 dimensional system. For force free granular system, initially the energy of the system cools down but the density is uniform throughout. This stage is called Homogeneous Cooling State (HCS) [26]. But, this state is unstable and at later stages clusters develop and the system continues to lose energy in Inhomogeneous Cooling State (ICS) $[7,8]$. For a heated granular system during early stages, for a particular value of coefficient of restitution for both force free and heated granular gas evolve in same fashion. But at later stages, the force free granular gas continues to cool, whereas heated granular gas settles to a steady state. During steady state, the kinetic energy of the system becomes independent of time. Further, it is clear that, as the value of $\epsilon$ is increased i.e. the system tends towards elastic limit, the value of steady state energy $E_{s s}$ of the system increases. For $\epsilon=0.80, E_{s s}$ is approximately $1.03 \times 10^{-3}$ and for $\epsilon=0.90, E_{s s}$ is approximately $2.02 \times 10^{-3}$. Also, the time at which the system reaches steady state also increases with increasing the value of $\epsilon$. For $\epsilon=0.80$, after approximately 50 collisions per particle have occurred steady state is reached and for $\epsilon=0.90$, steady state is reached after approximately 100 collisions per particle have occurred. In Fig.2, time evolution of the normalised kinetic energy of granular gas is shown on $\log$-log scale for 3 dimensional system. Similar results are observed for 3 dimensional system as 
well.

In Fig.3, we plot the normalised velocity autocorrelation function $\bar{A}\left(\tau_{w}, \tau\right)=A\left(\tau_{w}, \tau\right) / A\left(\tau_{w}, \tau_{w}\right)$ versus $\tau$ for $\epsilon=0.80$ and $\epsilon=0.90$ for 2-dimensional system on linearlog scale and in Fig.4 same is plotted for 3-dimensional system. A comparison study was done of these autocorrelation graphs with the normalised autocorrelation graphs obtained for force free granular gas Ref [8]. For force free gas, the decay of $\bar{A}\left(\tau_{w}, \tau\right)$ becomes slower for large values of waiting time which is referred to aging property of granular gas. Further, it is seen that the decay of $\bar{A}\left(\tau_{w}, \tau\right)$ is slower than exponential function in HCS which is due to correlations that build in the system prior to ICS. In the case of heated granular gas it is observed that at early stages when the system cools down like a force free gas, correlations build up in the system and $\bar{A}\left(\tau_{w}, \tau\right)$ decays slower than exponential. Also, there is an explicit dependence of $\bar{A}\left(\tau_{w}, \tau\right)$ on both $\tau$ and $\tau_{w}$ showing aging property. When steady state is reached, $\bar{A}\left(\tau_{w}, \tau\right)$ become independent of $\tau_{w}$ and does not show aging. However, the rate of decay of $\bar{A}\left(\tau_{w}, \tau\right)$ is slow showing presence of correlations in the system. On comparing Fig.3a and Fig.3b with Fig.4a and Fig.4b respectively it is observed that these velocity correlations are more pronounced in 2 dimensional system as compared to 3 dimensional system.

\section{Conclusion}

Aging behaviour of the velocity autocorrelation function is studied for uniformly heated granular gas. The results are compared with existing results for a force free granular gas. It is observed that initially $\bar{A}\left(\tau_{w}, \tau\right)$ depends on both $\tau$ and $\tau_{w}$ independently but after steady state is reached $\bar{A}\left(\tau_{w}, \tau\right)$ becomes independent of $\tau_{w}$. Even in the steady state, the decay of $\bar{A}\left(\tau_{w}, \tau\right)$ is slower than exponential. This shows existence of velocity correlations in uniformly heated granular gas even after steady state is reached. These correlations are less pronounced in $3 \mathrm{di}$ mensional system.

\section{Acknowledgements}

S.K. acknowledges the computational facilities provided for this work by Department of Physics, Jamia Millia Islamia, New Delhi, India. S.K. also acknowledges University Grants Commission, India for financial support.

\section{References}

[1] H. M. Jaeger, S. R. Nagel, and R. P. Behringer, Rev. Mod. Phys. 68, 1259 (1996).

[2] I. S. Aranson and L. S. Tsimring, Rev. Mod. Phys. 78, 641 (2006).
[3] A. Kudrolli, M. Wolpert, and J. P. Gollub, Phys. Rev. Lett. 78, 1383 (1997).

[4] L. P. Kadanoff, Rev. Mod. Phys. 71, 435 (1999).

[5] T. Pöschel and S. Luding, Granular Gases, in: Lecture Notes in Physics Vol. 564 (Springer, Berlin, 2001).

[6] T. Pöschel and N. V. Brilliantov, Granular Gas Dynamics, in: Lecture Notes in Physics Vol. 624 (Springer, Berlin, 2003).

[7] N. V. Brilliantov and T. Pöschel, Kinetic Theory of Granular Gases (Oxford University Press, Oxford, 2004).

[8] S. R. Ahmad and S. Puri, Phys. Rev. E 75, 031302 (2007).

[9] I. Goldhirsch and G. Zanetti, Phys. Rev. Lett. 70, 1619 (1993).

[10] S. Kumari and S. R. Ahmad, AIP Conference Proceedings 1728, 020680 (2016).

[11] S. K. Das and S. Puri, EuroPhys. Lett. 61, 749 (2003).

[12] R. D. Wildman and D. J. Parker, Phys. Rev. Lett. 88, 064301 (2002).

[13] K. Feitosa and N. Menon, Phys. Rev. Lett. 88, 198301 (2002).

[14] O. Zik, D. Levine, S. G. Lipson, S. Shtrikman and J. Stavans, Phys. Rev. Lett. 73, 644 (1994).

[15] L. Bocquet, W. Losert, D. Schalk, T. C. Lubensky and J. P. Gollub , Phys. Rev. E 65, 011307 (2001).

[16] A. Snezhko, I. S. Aranson and W. K. Kwok, Phys. Rev. Lett. 94, 108002 (2005).

[17] I. S. Aranson and J. S. Olafsen, Phys. Rev. E 66, 061302 (2002).

[18] E. Ben-Naim and P. L. Krapivsky, Phys. Rev. E 66, 011309 (2002).

[19] T. P. C. van Noije and M. H. Ernst, Granular Matter 1, 57 (1998).

[20] D. R. M. Williams and F. C. MacKintosh, Phys. Rev. E 54, R9 (1996); D. R. M. Williams, Physica A 233, 718 (1996).

[21] Sung Joon Moon, M. D. Shattuck, and J. B. Swift, Phys Rev. E 64, 031303 (2001).

[22] Awadhesh Kumar Dubey, Anna Bodrova, Sanjay Puri and Nikolai Brilliantov, Phys Rev. E 87, 062202 (2013).

[23] G. Peng and T. Ohta, Phys. Rev. E 58, 4737 (1998).

[24] J. M. Montanero and A. Santos, Granular Matter 2, 53 (2000).

[25] D. C. Rapaport, The Art of Molecular Dynamics Simulation, Second edition (Cambridge University Press, Cambridge, 2005).

[26] P. K. Haff, J. Fluid Mech. 134, 401 (1983). 